Criterion of Validity." Higher Education 20(2):135-142.

Seldin, Peter. 1989. "How Colleges Evaluate Professors." American Association for Higher Education Bulletin 41(7)(March):3-7.

Scherr, Frederick C., and Susan S. Scherr. 1990. "Bias in Student Evaluations of Teacher Effectiveness." Journal of Education for Business 65(8):356-358.

Smith, Sharon P., and Daniel P. Kinney. 1992. "Age and Teaching Performance." Journal of Higher Education 63(3):282302 .

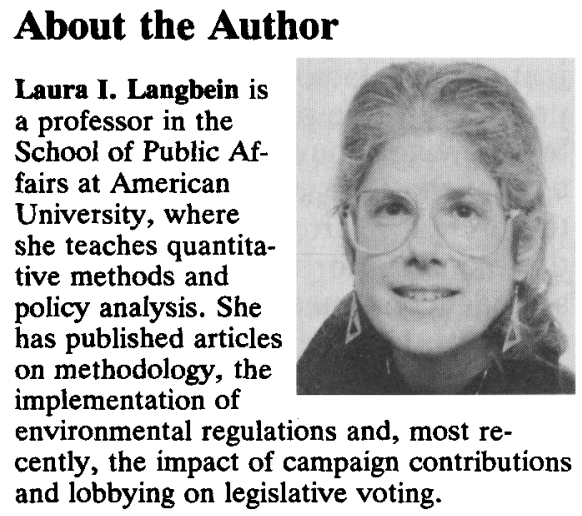

About the Author

chool of Public A

policy analysis. She

published articles

implementation of

cently, the impact of campaign contributions

and lobbying on legislative voting.

\title{
Teaching Research Methods Using Appropriate Technology*
}

\author{
Allan McBride, Grambling State University
}

Undergraduate and graduate-level courses in social science research methods are widely avoided and maligned by students while fàculty members who are required, or who choose, to teach these courses often suffer from poor student evaluations. The reasons for this situation are related to the nature of the material, which leaves little opportunity for students to apply the knowledge they have gained in other courses in their major; at least students believe this to be the case. Additionally, students are required to master the language of scientific methods, with specific and technical definitions; to understand scientific and experimental notation; and to comprehend the difficult area of probability theory and its relation to sampling, all matters for which students see little or no purpose. Yet faculty recognize that students who are to be well-informed citizens, or to attend graduate school, or to seek professional employment upon graduation need to master some of these skills to contribute successfully to their

\footnotetext{
*The ability to conduct the type of research described in this paper has been enhanced by National Science Foundation Instrumentation and Laboratory Enhancement grant \#USE-9251254. Grants of this sort are available to undergraduate institutions to help them improve laboratory facilities for undergraduate uses.
}

communities and to their professional lives.

Further contributing to this disturbing scenario is the evidence that many undergraduate, and possibly some graduate, students lack cognitive sophistication. Hudak and Anderson (1990) report that as many as $\mathbf{5 0 \%}$ of undergraduate students are not capable of abstract thinking. Whether this is evidence of a flawed theory of human development, a failure of secondary and elementary education, or the result of more widely available postsecondary education, is unclear. However, it does suggest that university faculty must be more sensitive to students' limitations and design their courses to take advantage of the capabilities that they have when they arrive on campus.

I have taught research methods for eight years at an open admissions HBCU (Historically Black College/University) both at the graduate and undergraduate levels and have had to deal with all the problems mentioned above, writ large in comparison to faculty who have the luxury of teaching in more selective universities. Even so, I think it is possible to engage students in the research process, even in a single-semester course, so that they can experience the pleasure and excitement of conducting re- search while learning its basic principles.

It is my purpose in writing to share my experiences with other faculty concerning some practical, hands-on methods for teaching a course in research methods-methods that are suitable for students who are at what Piaget referred to as the concrete operations stage of development. The approaches that I discuss in this paper are suitable for both undergraduate and graduate classes, and can be used successfully even where student research sophistication is quite low.

\section{Appropriate Technology for Research Methods}

The term "appropriate technology" was coined in the 1970 s to describe energy generation methodologies that were readily accessible to a broad sector of the population, were relatively inexpensive, and were easy to employ. I use the term in the same sense to suggest that the methods of research can be accessible, inexpensive, and easy to use, particularly with the development of personal computers (though not solely for that reason). Below I identify some approaches to research that meet these three criteria. 
The research approach that I describe below, as intimated above, can be successful at institutions with limited resources, in both libraries and technical support. At my institution, our technical resources are limited to a small computer lab (six computers on a LAN) and local access telephone lines. We do have access to a mainframe computer, but the lab is better suited to our purposes.

\section{Developing a Topic}

The first step in undertaking a research project is to identify a manageable topic. For undergraduate, and for many graduate, students this is a difficult and intimidating task. Topic selection can be made more manageable by two relatively simple techniques, neither of which is particularly innovative: first, we usually devote a class session during the first week of school to identifying potential research projects. In one graduate public administration research methods class, for example, the list included homelessness, shelters for the mentally impaired, solid waste, drug rehabilitation programs, AIDS and sex education programs for teenagers, and negative campaign advertising. The students were allowed to select which topic they preferred to work with; some adjustments were later made as students changed their minds about their interests. I played an active role in the topic adoption process to insure that topics were amenable to empirical research and manageable in the time available.

Second, as the list of topics was developed students selected the project that they found most attractive, which consequently broke the class into work teams. The ability to work in groups is valuable to almost any employer, and weaker students will benefit from the skills and leadership of the stronger students. The stronger students, in turn, benefit by developing their own understanding through explaining difficult concepts and procedures to others.

Group work is not without its drawbacks, however, as many of the more diligent group members will resent those who do not participate at satisfactory levels. This problem can be partially addressed by allowing group members to evaluate each other at various stages of the project. I provide them with three criteria for those evaluations: attendance at meetings held outside the class, level of preparation undertaken by group members for those meetings, and level of participation in the meetings. I also allow the groups to meet periodically during class time.

Second, with about 25 students in the class each group had 4 to 5 members, making them small enough to allow members to become well acquainted (this, of course, can be an advantage and disadvantage. I receive regular reports from members about others in their group who create problems in one manner or another. I listen and encourage them to work out their difficulties as best they can, reminding them of the opportunity for self-evaluation later), and large enough to overcome the fear that they will not be able to manage the project alone.

\section{The Literature Review}

Ideally, the topic should be chosen after a careful review of relevant literature, a point we discuss during class sessions. However, the time constraints associated with a single-semester class make that approach impractical. Yet students, and the project, can benefit in a number of ways from a limited review of academic journals. Students may be able to identify useful theories and models, they can gain insights into the type of research design that may be most appropriate for the research that they are conducting, and they should find relevant measures of the variables that they are planning to use.

Again it would be most valuable for each student to undertake a thorough investigation of the literature themselves. However, if the number of sources is limited to two unique journal articles per student, students are more likely to make a relatively serious effort to read these fully and address issues related to their project. I provide them with a list of questions related to research methods that they may address in the course of the review: What is the theory or model being used by the author(s)? What hypotheses do the authors develop from that theory? What sort of research design do they use to test the hypothesis? In regard to this question they are to address issues related to the nature and size of the sample, where the research was conducted, the measures that the authors employed and whether the research is survey, experimental, one-shot, longitudinal, and so forth. As much as possible they should then discuss the type of analysis the authors are undertaking.

The data-analysis section of the articles reviewed by the students provides special challenges. Novice researchers will stumble when discussing complex and technical statistical analyses; therefore I encourage them to choose articles that have relatively simple data analysis and to clear their choice of articles with me before beginning to read. Finally, they should report the findings of the study, whether the findings support the hypotheses, and what implications the research has for their own project.

This portion of the project has been undertaken by each student independently and therefore grades can be assigned to students based upon their own work. Since each student is required to find unique articles, the group will have access to as many as 8 to 12 relevant articles, mostly from recent research journals.

\section{The Research Design}

This critical aspect of the research project presents additional problems for the novice. All the research that $I$ have conducted in this manner has been of a survey nature (though I feel certain that experimental research projects would be equally successful) but there are a variety of approaches to survey research, each of which is most appropriate in answering certain kinds of research questions. 
The relationship between the design and the research question is too subtle for many students to grasp at first. While it is desirable to make sure that each group clearly links their design to their topic, this is often impracticable. My goal, therefore, is to make them tackle the issue at least by thinking about the same sorts of questions for their own project that they addressed in the literature review mentioned above. In class and in our readings they are introduced to one-shot designs, longitudinal and panel studies, comparison and contrasting samples, sampling, and populations. Here again it may be necessary for the instructor to provide as much guidance as is possible in meetings with the group, without stifling their own creative impulses.

Research design development is conducted "en group," with the group making a presentation to the class about how they plan to conduct their research. Other class members provide comments and constructive criticism after the presentation. While they should be reading the materials for the literature review by early in the semester, the first portion of the project to be completed will be the research design. It is the first opportunity they have, then, to evaluate each other's performance using the criteria described above.

\section{The Questionnaire}

While students are beginning the literature review and research design, with an early due date to avoid the demands of end-of-semester grading, they also continue to attend class where the concepts and procedures associated with empirical research are being discussed and detailed. Within several weeks after the start of the semester, we begin to discuss data collection and measurement and the practical aspects of questionnaire design. Babbie (1990, chap. 7) provides an excellent guide to question construction, addressing grammar and vocabulary issues as well as issues related to measurement; he also provides valuable guidance on the issue of the design of the questionnaire itself.

With this discussion in mind, the groups should begin to design their questionnaires. This portion of the project is undertaken by the group as a whole. If and when they encounter problems or uncertainty they are encouraged to schedule a time to meet when I can be with them to insure that they are progressing satisfactorily. At a point near the middle of the semester, the groups will each make an informal presentation to the class of a clean copy of the questionnaire. Class members, including the instructor, will provide constructive criticism of the layout, items, and relevance of the questionnaire for the stated project. This achieves the goals of providing class members the opportunity to review written work and criticize it, while allowing the research group to gain the advantage of useful comments. They may use these comments later to improve the quality of their questionnaire. This may be done by either allowing them to return to the drawing board and resubmit a final version later or by grading the product as is and allowing individual members the chance to revise the protocol independently, using the class critique as the basis for their improvements, for extra credit.

Typically two grades are given on the questionnaire: the first is assigned by the group members to one another, using the criteria listed above, and the second is assigned by me to each group member based upon the final product. Depending upon the ultimate use for the project (for example if students have expressed an interest in making a presentation at some conference) I may take the responsibility for the final version of the questionnaire myself.

\section{The Sample}

At this point it is useful to mention some variations in these procedures that may be useful if the class is a relatively small one, say under 15 students. In that case, instead of dividing into groups we may undertake a single project, and because we are all working in the same direction we may be able to get further into the project. When multiple projects are underway simultaneously we normally do not get much further than the design and the questionnaire.

With fewer students it may be possible actually to design a small sample and require each class member to interview a reasonable number of respondents (10 to 15 per student). Depending upon the nature of the research, a satisfactory pedagogical sample can be drawn from the telephone directory, the city directory, enrollment lists from the registrar's office, or voter registration lists. I have also used to advantage more specialized lists including a listing of the classes offered during a semester to sample from classrooms, the listing of the top 500 U.S. corporations of the Fortune 500 to draw names of corporate executives, and daily police reports in the local newspaper to identify persons who had been arrested for driving while intoxicated. Many organizations maintain active-member rosters that could be used equally well to develop a sample of some special population.

Once this roster is in hand, access to a personal computer provides the opportunity to design a simple quasi-random sample of persons who may be contacted (SPSSPC has a sampling command, as do other data-analysis software packages).

\section{Data Collection}

Once a sample has been identified students will have the opportunity to interview and collect data, time permitting. I have used several methods of data collection, including telephone, mail, and the personally administered questionnaire. Each method has advantages and will be discussed below. Obviously the telephone is a clear example of appropriate technology, as many professional researchers use telephones to conduct public opinion polls. The advantage for the classroom teacher is that use of the telephone system in the department allows the instructor to supervise 
the interviewers directly. MicroCase analysis system provides the capability to design a template that can be used to administer the questionnaire and to record responses. For those who have computer labs with telephones, this provides opportunities to avoid the boredom of coding.

Because this is a student project, we normally will spend one evening in the department offices making the first phone calls. This gives each student the chance to make two or three calls while I wander around providing comments on their technique. We will usually precede this session with one-onone practice in the classroom, during which time we stress the importance of staying with the dialogue and being comfortable with the protocol. Inevitably, though, some students will come underprepared, providing immediate and direct evidence of the virtue of preparation.

Mailing questionnaires has wellknown advantages, coupled with the serious drawback of low return rates. The advantage for a class project is that the questionnaire can be handled without the problems associated with interviewer training. The primary shortcoming of the mailed protocol from the instructor's perspective is that the returns are going to be coming directly to your mailbox. While it is gratifying to see them, it probably means spending some time organizing and recording their arrival, even assuming that the students will perform all the necessary coding operations.

The personally administered questionnaire has a number of advantages for a project of this sort; ease of administration is the chief one. There is no need to train interviewers, and the questionnaire is usually easier to design because there is no need for lengthy interviewer instructions. There are two problems of which the instructor should be aware: first, if students are to hand out these questionnaires, how will they choose their respondents? If the project is strictly pedagogical, then it may not matter if they use a sample of convenience. If there are plans to make use of the data set in other projects, then this is a serious deficiency. The second problem is one of return rate; if the questionnaire is left to be filled out, if may suffer from the same problem that plagues mailed questionnaires.

For student projects there is a reasonable solution to these problems: use a bloc sample design based upon university-wide class offerings and administer the questionnaire to an entire class at once. If faculty members from other disciplines are contacted in advance and informed of the nature of the project, most prove to be cooperative. There are many suitable research questions dealing with student concerns where this approach is useful. I have undertaken two such projects: one dealt with stopand-search issues in a southern university town and the second dealt with educational outcomes at two southern universities. Students found both of these topics to be of great interest.

\section{Coding and Analysis}

The coding stage is undoubtedly the least attractive aspect of research: mind-numbing, repetitive work that nonetheless is extremely important. Finding competent people who can be trusted to complete this onerous task is difficult, but there are two viable alternatives. As do many researchers, I incorporate the coding scheme into the questionnaire; as they are returned, or completed, students may take a few moments to carry out the coding on the spot. Alternatively, half a class period is usually sufficient to complete the coding on 200 or so protocols, if the students are broken into work teams of two or three. This may result in a few more coding related errors, but most of these can be located relatively easily during the analysis stage. Even troublesome openended items can be handled in a classroom setting. As students code I ask them to identify the unique responses to open-ended items that I write on the board, numbering them as they appear. As they encounter identical responses the numbered categories are already visible and may be easily coded.

By the time the data are all returned the semester is usually in its last week or so, making analysis an impossibility for the students. Since these are usually small data sets, 150 to 200 respondents, I may be able to do the data entry myself and make a presentation in class based upon frequencies and crosstabulations. Several alternatives remain for the data in the future. Students may avail themselves of them to write papers for other classes, they may be used in statistics classes as demonstration data, or they may be used by the highly motivated student to present a paper at a local conference.

\section{Examples}

I started teaching research methods when I arrived at Grambling in 1985 and early on began to experiment with direct experience for students. It was probably not until 1987 that we carried out our first complete project, however. In that year we conducted a nationwide mailed survey of administrators in state government agencies and compared their responses with those of administrators from large corporations. This turned into a presentation at a regional conference for one of the class participants. The next year, we did a telephone interview of local residents and measured their satisfaction with local government services. Class members did some preliminary data analysis that year and presented their findings at a city council meeting, resulting in favorable press coverage in the local newspaper.

A more recent project did not fare quite as well. The topic was a program evaluation of the local alcohol abuse treatment program. The students developed a questionnaire and drew a sample from police reports in the local newspaper. That, however, turned out to be a poor listing of potential respondents as most of the addresses were incorrect and we were able to collect only a few questionnaires. Even so, students were able to ex- 
perience first-hand some of the difficulties of research and sampling.

Being a member of the faculty at an HBCU presents its own unique research opportunities; as noted above, one semester we compared stop-and-search experiences between black and white students using samples from our campus as well as a nearby "white" university. I also directed a class project in which we compared educational outcomes and experiences between these same two universities. The latter paper has been used by a graduate student for presentation at a local political science conference.

\section{Conclusion}

Not all projects are equally beneficial, but it is my belief that stu- dents gain considerably from this hands-on approach to research. While grades may not be higher, the experience of designing research, composing a questionnaire, collecting and eventually analyzing data, cannot but help students to increase their understanding of the social scientific process. Evaluating local programs, using available technology for contacting respondents, drawing samples, and analyzing data put research of this type within the reach of even the smallest of departments. As a student I was not given the opportunity to conduct primary research of this sort until I was working on my own dissertation. Offering undergraduate and graduate students this opportunity early in their academic careers will be of benefit not only to them but to the discipline as well.

\section{References}

Babbie, Earl. 1990. Survey Research Methods, 2nd ed. Belmont, CA: Wadsworth Publishing.

Hudak, Mary, and David Anderson. 1990. "Formal Operations and Learning Style Predict Success in Statistics and Computer Science Courses." Teaching of Psychology: 231-34.

\footnotetext{
About the Author

Allan McBride is an associate professor at Grambling State University. His research interests include political communication, political behavior, and political culture. $\mathrm{He}$ is currently investigating popular television programming for evidence of political culture content.
} 